\title{
INVARIANT MEANS ON A CLASS OF VON NEUMANN ALGEBRAS
}

\author{
BY
}

P. F. RENAUD

ABSTRACT. For $G$ a locally compact group with associated von Neumann algebra $V N(G)$ we prove the existence of an invariant mean on $V N(G)$. This mean is shown to be unique if and only if $G$ is discrete.

1. Definitions and preliminaries. Throughout we follow in general the notation of [3]. Let $G$ be a locally compact group with identity $e$. Let $L_{1}(G)$ be the group algebra of $G$ with respect to left Haar measure and let $C^{*}(G)$ be the enveloping $C^{*}$-algebra of $L_{1}(G)$. Denote by $B(G)$ the dual space of $C^{*}(G)$. Then $B(G)$ may be realized as the space of all finite linear combinations of continuous positive-definite functions on $G$. With the dual norm and pointwise multiplication, $B(G)$ is a commutative Banach algebra-the Fourier-Stieltjes algebra of $G$. The closed subalgebra $A(G)$ generated by elements with compact support is called the Fourier algebra of $G$. Let $P=\{u \in A(G): u$ is positive definite and $\|u\|=u(e)=$ $1\}$.

For $x$ a complex-valued (a.e. defined) function on $G$, denote by $\check{x}, \tilde{x}$ the functions

$\check{x}(g)=x\left(g^{-1}\right), \quad \tilde{x}(g)=\overline{x\left(g^{-1}\right)}$ where - denotes complex conjugation.

If $f \in L_{1}(G)$, denote by $L_{f}$ the bounded operator on $L_{2}(G)$ defined by $L_{f} x=$ $f * x$ (where * denotes convolution).

An important property of $A(G)$ is that it is precisely the set of functions of the form $x * \tilde{y}$ where $x, y \in L_{2}(G)$ [3, p. 218].

Let $V N(G)$ denote the von Neumann algebra on $L_{2}(G)$ generated by the operators $L_{f}$ where $f \in L_{1}(G)$. Then $V N(G)$ may be regarded as the dual space of $A(G)$ under the map $\langle T, u\rangle \doteq(T x, y)$ if $u \in A(G)$ with $u=\tilde{y} * \tilde{x}$. In particular, the $w^{*}$ - and weak operator topologies on $V N(G)$ coincide. For $u \in A(G), T \in$ $V N(G)$ define the operator $u T \in V N(G)$ by

$$
\langle u T, v\rangle=\langle T, u v\rangle, \quad v \in A(G) .
$$

It follows readily that $V N(G)$ is an $A(G)$-module and that $\|u T\| \leq\|u\|\|T\|$.

A linear functional $m$ on $V N(G)$ is called a mean if

Received by the editors June 28, 1971. $43 \mathrm{~A} 40$.

AMS 1970 subject clas'sfications. Primary 22D15, 43A15; Secondary 22D25, 43A07,

Key words and phrases. Locally compact group, von Neumann algebra, Fourier algebra, invariant mean. 
(i) $m(T) \geq 0$ if $T \geq 0$, and

(ii) $m(I)=1$ where $I$ is the identity operator.

$m$ is called an invariant mean if it satisfies (i), (ii), and

(iii) $m(u T)=m(T)$ for all $T \in V N(G), u \in P$, or equivalently

(iii') $m(u T)=u(e) m(T)$ for all $T \in V N(G), u \in A(G)$.

A linear functional $m$ satisfying (iii) will be called invariant.

If $G$ is Abelian with dual group $\hat{G}$ then $A(G)$ is isomorphic to $L_{1}(\hat{G})$ and $V N(G)$ to $L_{\infty}(\hat{G})$. The existence of invariant means on $L_{\infty}(\hat{G})$ is guaranteed since $\hat{G}$ is Abelian and hence amenable (see [4] for a comprehensive account of amenability). The main purpose of this paper is to show that for every locally compact group $G$, there exists an invariant mean on $V N(G)$ and that this mean is unique if and only if $G$ is discrete. In a certain sense therefore, the "dual structures" of an arbitrary group enjoy the property of amenability. For convenience of exposition we treat the discrete case separately.

2. The discrete case.

Theorem l. Let $G$ be a discrete group. Then there exists a unique invariant mean on $V N(G)$.

Proof. The function $\delta_{e}$ defined by $\delta_{e}(g)=1$ if $g=e$ and 0 otherwise is in $L_{2}(G)$ and since $\delta_{e} * \widetilde{\delta}_{e}=\delta_{e}$, then $\delta_{e}$ is also in $A(G)$. Define the linear functional $m$ on $V N(G)$ by

$$
m(T)=\left\langle T, \delta_{\boldsymbol{e}}\right\rangle=\left(T \delta_{\boldsymbol{e}}, \delta_{\boldsymbol{e}}\right) .
$$

It is immediate that $m$ is a mean. We show that $m$ is invariant.

Let $T \in V N(G), u \in P$. Since $u(e)=1$ we have $u \delta_{e}=\delta_{e}$ and hence

Thus $m$ is invariant.

$$
m(u T)=\left\langle u T, \delta_{e}\right\rangle=\left\langle T, u \delta_{e}\right\rangle=\left\langle T, \delta_{e}\right\rangle=m(T) .
$$

To show uniqueness suppose that $m^{\prime}$ is an invariant mean on $V N(G)$. Let $T \in V N(G), v \in A(G)$. We have

$$
\left\langle\delta_{e} T, v\right\rangle=\left\langle T, \delta_{e} v\right\rangle=\left\langle T, v(e) \delta_{e}\right\rangle=v(e)\left\langle T, \delta_{e}\right\rangle=v(e) m(T)=\langle m(T) I, v\rangle
$$

and hence $\delta_{\boldsymbol{e}} T=m(T) I$. But then since $m^{\prime}$ is invariant and $\delta_{\boldsymbol{e}} \in P$,

$$
m^{\prime}(T)=m^{\prime}\left(\delta_{\boldsymbol{e}} T\right)=m^{\prime}(m(T) I)=m(T),
$$

which proves uniqueness.

Remarks. 1. It is easy to show that in this case, $m$ is a faithful normal trace on $V N(G)$ so that $V N(G)$ is a finite von Neumann algebra.

2. If $G$ is Abelian and discrete, $\hat{G}$ is compact and it is well known that in this case $L_{\infty}(\hat{G})$ has a unique invariant mean, namely the Haar integral.

3. The general case. A useful aid to the study of amenability is the concept of "convergence to invariance". We introduce a similar idea and show its relationship to the existence of invariant means. 
Definition 2. A net $\left\{u_{\alpha}\right\} \subset P$ is called $w$ - (norm) convergent to invariance if for all $v \in P$,

$$
w-\lim \left(u_{a} v-u_{a}\right)=0, \quad \text { i.e. } \quad \lim \left\langle u_{a} v-u_{a^{\prime}} T\right\rangle=0
$$

for all $T \in V N(G)$.

$$
\left(\lim \left\|u_{a} v-u_{a}\right\|=\right.
$$

The following proposition proves the existence of such nets. For compact groups it is proved in [2].

Proposition 3. Let $\left\{V_{a}\right\}$ be a neighbourbood basis at $e$ and for each $a$, choose $u_{a} \in P$ such that spt $u_{\alpha}$ (support of $u_{a}$ ) $\subset V_{a}$. Directed by inclusion, $\left\{u_{\alpha}\right\}$ is norm convergent to invariance.

Proof. Fix $v \in P, \epsilon>0$. Choose $v^{\prime} \in P$ with compact support $K$ and such that $\left\|v-v^{\prime}\right\|<\epsilon / 2$. By [3, p. 208], there exists some $u \in A(G)$ with $u(g) \equiv 1$ on $K$. Then $(u-v)(e)=0$ so that by [3, p. 229] we can find an element $w \in A(G)$ with $\|u-v-w\|<\epsilon / 2$ and $w$ identically zero on some neighbourhood $U$ of $e$. Now if $V_{\alpha} \subset U \cap K$ then $u_{\alpha} u=u_{\alpha}$ and $u_{\alpha} w=0$. Hence for such $\alpha$ 's,

$$
\begin{aligned}
\left\|u_{a} v-u_{a}\right\| & \leq\left\|u_{a}\left(v-v^{\prime}\right)\right\|+\left\|u_{a} v^{\prime}-u_{a}\right\| \\
& <\epsilon / 2+\left\|u_{a} v^{\prime}-u_{a}\right\|=\epsilon / 2+\left\|u_{a}\left(v^{\prime}-u-w\right)\right\| \\
& \leq \epsilon / 2+\left\|v^{\prime}-u-w\right\|<\epsilon .
\end{aligned}
$$

Hence $\left\{u_{a}\right\}$ is norm convergent to invariance.

Using this result we can prove

Theorem 4. Let $N$ denote the set of invariant means on $V N(G)$. Then $N$ is a nonempty convex set.

Proof. We consider $A(G)$ as a subspace of $V N(G)^{*}$ in the usual way. By Proposition 3 there exists a net $\left\{u_{a}\right\} \subset P, w$-convergent to invariance. Since the unit ball of $V N(G)^{*}$ is $w^{*}$-compact, $\left\{u_{a}\right\}$ has a $w^{*}$-accumulation point $m$. Let $\left\{u_{\beta}\right\}$ be a subnet $w^{*}$-convergent to $m$. Each $u_{\beta}$ is clearly a mean so that $m$ is a mean. If $u \in P, T \in V N(G)$ then

$$
m(u T)=\lim \left\langle u_{\beta}, u T\right\rangle=\lim \left\langle u_{\beta} u, T\right\rangle
$$

and since $\left\{u_{\beta}\right\}$ is $w^{*}$-convergent to invariance,

$$
\lim \left[\left(u_{\beta} u, T\right\rangle-\left\langle u_{\beta}, T\right\rangle\right]=0
$$

so that

$$
m(u T)=\lim \left\langle u_{\beta}, T\right\rangle=m(T)
$$

and $m$ is an invariant mean. Hence $N$ is nonempty. That $N$ is convex is immediate.

The following proposition gives a useful distinction between discrete and nondiscrete groups. 
Proposition 5. Let $m$ be an invariant mean on $V N(G)$. If $m \in A(G)$ then $G$ is discrete.

Proof. Suppose that $m \in A(G)$ so that there exists some $u \in A(G)$ such that $m(T)=\langle u, T\rangle$ for all $T \in V N(G)$. Then $\langle u, T\rangle=m(T)=m(v T)=\langle v T, u\rangle=\langle T, v u\rangle$ for all $T \in V N(G), v \in P$. It follows that $u=v u$ for all $v \in P$. If now $G$ is nondiscrete we can choose $g \in G$ such that $u(g) \neq 0$ and $v \in P$ such that $v(g)=0$. This contradicts the fact that $u=v u$. Hence $G$ is discrete.

We now consider the problem of uniqueness. Specifically we want to show that if $G$ is nondiscrete then there is more than one invariant mean on $V N(G)$. We commence by establishing some properties of the dual space $V N(G)^{*}$.

In [1] Arens showed that, given a Banach algebra $B$, it is possible to define a multiplication on $B^{* *}$ which extends multiplication on $B$. In the case where $B=$ $A(G)$, the idea is as follows, for $m \in V N(G) *, T \in V N(G)$ define $m \circ T \in V N(G)$ by $\langle m \circ T, u\rangle=\langle m, u T\rangle$ for all $u \in A(G)$.

For $m, n \in V N(G)^{*}$, define $m \circ n \in V N(G) *$ by $\langle m \circ n, T\rangle=\langle m, n \circ T\rangle$ for all $T \in V N(G)$.

This operation $\circ$ makes $V N(G)^{*}$ a Banach algebra where also $\|m \circ n\| \leq$ $\|m\| n \|$ for all $m, n \in V N(G)^{*}$.

Lemma 6. Let $m \in V N(G) *$. Then $m$ is invariant if and only if $m=v \circ m$ for all $v \in P$.

Proof. For $T \in V N(G)$ we have

$$
\langle v \circ m, T\rangle=\langle v, m \circ T\rangle=\langle m \circ T, v\rangle=\langle m, v T\rangle
$$

and the result follows.

Corollary 7. Let $m, n \in V N(G) *$. If $m$ is invariant then so is $m \circ n$.

Proof. For if $v \in P$ then

$$
v \circ(m \circ n)=(v \circ m) \circ n=m \circ n \text {. }
$$

Let $H$ be a normal, compact subgroup of $G$. Eymard [3, p. 217] has shown that $A(G / H)$ may be identified with the subalgebra of $A(G)$ consisting of all functions which are constant on the cosets of $H$. The adjoint of this identification is a homomorphism $\pi$ of $V N(G)$ onto $V N(G / H)$. Similarly we may identify $L_{2}(G / H)$ with the subspace $M$ of $L_{2}(G)$ of all functions constant on the cosets of $H$. For $T^{\prime} \epsilon$ $V N(G / H)$ define the operator $\rho T^{\prime}$ on $L_{2}(G)$ by $\rho T^{\prime} x=T^{\prime} x$ if $x \in M$ and $\rho T^{\prime} x=0$ if $x \in M^{\perp}$. It follows readily that $\rho T^{\prime} \in V N(G)$ and that $\rho$ is an isometric embedding of $V N(G / H)$ into $V N(G)$.

For $u \in A(G), f\left(T^{\prime}\right)=\left\langle u, \rho T^{\prime}\right\rangle$ is an ultraweakly continuous linear functional on $V N(G / H)$ so that $f\left(T^{\prime}\right)=\left\langle u^{\prime}, T^{\prime}\right\rangle$ for some uniquely defined $u^{\prime} \in A(G / H)$.

With these results we can prove

Proposition 8. Let $H$ be a normal, compact subgroup of $G$. Let $m$ be an invariant mean in $V N(G)^{*}$ and $n^{\prime}$ an invariant element in $V N(G / H)^{*}$. Let $n=\pi^{*} n^{\prime}$. Then $m \circ n$ is invariant and $\|m \circ n\|=\left\|n^{\prime}\right\|$. 
Proof. By Corollary 7, we know that $m \circ n$ is invariant. Also since

$$
\|m \circ n\| \leq\|m\|\|n\|=\left\|\pi^{*} n^{\prime}\right\| \leq\left\|n^{\prime}\right\|
$$

it suffices to prove that $\|m \circ n\| \geq\left\|n^{\prime}\right\|$.

Fix $\epsilon>0$ and choose $T^{\prime} \in V N(G / H)$ such that $\left\|T^{\prime}\right\|=1$ and $\left|\left\langle n^{\prime}, T^{\prime}\right\rangle\right| \geq$ $\left\|n^{\prime}\right\|-\epsilon$. If $u \in A(G), v^{\prime} \in A(G / H)(C A(G))$ then

$$
\begin{aligned}
\left\langle\pi\left(u \rho T^{\prime}\right), v^{\prime}\right\rangle & =\left\langle u \rho T^{\prime}, v^{\prime}\right\rangle=\left\langle\rho T^{\prime}, u v^{\prime}\right\rangle=\left\langle v^{\prime} \rho T^{\prime}, u\right\rangle \\
& =\left\langle v^{\prime} T^{\prime}, u^{\prime}\right\rangle=\left\langle u^{\prime} T^{\prime}, v^{\prime}\right\rangle
\end{aligned}
$$

so that $\pi\left(u \rho T^{\prime}\right)=u^{\prime} T^{\prime}$.

Hence

$$
\begin{aligned}
& \left\langle n \circ \rho T^{\prime}, u\right\rangle=\left\langle n, u \rho T^{\prime}\right\rangle=\left\langle\pi^{*} n^{\prime}, u \rho T^{\prime}\right\rangle \\
& =\left\langle n^{\prime}, \pi\left(u \rho T^{\prime}\right)\right\rangle=\left\langle n^{\prime}, u^{\prime} T^{\prime}\right\rangle=u^{\prime}(e)\left\langle n^{\prime}, T^{\prime}\right\rangle
\end{aligned}
$$

so that $n \circ \rho T^{\prime}=\left\langle n^{\prime}, T^{\prime}\right\rangle$.

Therefore,

$$
\left|\left\langle m \circ n, \rho T^{\prime}\right\rangle\right|=\left|\left\langle m, n \circ \rho T^{\prime}\right\rangle\right|=\left|\left\langle m,\left\langle n^{\prime}, T^{\prime}\right\rangle I\right\rangle\right|=\left|\left\langle n^{\prime}, T^{\prime}\right\rangle\right| \geq\left\|n^{\prime}\right\|-\epsilon
$$

from which we deduce that $\|m \circ n\| \geq\left\|n^{\prime}\right\|$.

Corollary 9. If $H$ is compact and $V N(G / H) *$ admits more than one invariant mean then so does $V N(G) *$.

Proof. Let $m$ be an invariant mean on $V N(G)$. Define $\sigma: V N(G / H)^{*} \rightarrow$ $V N(G)^{*}$ by $\sigma\left(n^{\prime}\right)=m \circ \pi^{*} n^{\prime}$. By the previous proposition, $\sigma$ is an isometry from the subspace of invariant elements in $V N(G / H) *$ into the subspace of invariant elements in $V N(G) *$. Consequently, if $n_{1}^{\prime}, n_{2}^{\prime}$ are distinct invariant means in $V N(G / H) *$ then $\sigma\left(n_{1}^{\prime}\right), \sigma\left(n_{2}^{\prime}\right)$ are distinct invariant means in $V N(G) *$.

Finally we shall need

Proposition 10. Let $K$ be an open subgroup of $G$ and suppose that there are two distinct invariant means on $V N(K)$. Then there are two distinct invariant means on $V N(G)$.

Remark. For $G$ compact, $K$ a closed subgroup, this result was proved in [2, Theorem 6]. The proof carries over to noncompact groups and is included for completeness.

Proof. Let $\phi$ denote the restriction homomorphic map, $\phi: A(G) \rightarrow A(K)$. The map $\phi$ is bounded and since $K$ is open, $\phi$ is onto [3, p. 215]. It suffices to show that if $m^{\prime}$ is an invariant mean on $V N(K)$ then there exists an invariant mean $m$ on $V N(G)$ such that $m^{\prime}=m \mid \phi^{*} V N(K)$.

Let $m^{\prime}$ be an invariant mean on $V N(K)$. It follows from a general theorem on vector lattices [6, Theorem 4.3] that there exists a net $\left\{v_{a}^{\prime}\right\}$ of normalized positivedefinite functions in $A(K)$ such that $w^{*}-\lim v_{\alpha}^{\prime}=m^{\prime}$. For $V$ a neighbourhood of $e$ 
in $G$, choose $u_{V} \in P$ such that spt $u_{V} \subset V$. Let $v_{a, V}^{\prime}=v_{\alpha}^{\prime} u_{V} \mid H$ and observe that if $T \in V N(K)$ then for all $V$,

$$
\lim \left\langle v_{a, V}^{\prime}, T\right\rangle=\lim i v_{a}^{\prime}, u_{V}|H \cdot T\rangle=\left\langle m^{\prime}, u_{V} \mid H \cdot T\right\rangle=\left\langle m^{\prime}, T\right\rangle .
$$

Choose $v_{\alpha} \in P$ such that $\phi v_{\alpha}=v_{\alpha}^{\prime}$ and define $v_{\alpha, V}=v_{\alpha} u_{V}$. Let $m$ be a $w^{*}$-accumulation point of $\left\{v_{a, V}\right\}$. By the proof of Theorem $4, m$ is an invariant mean on $V N(G)$. We show that $m \mid \phi^{*} V N(K)=m^{\prime}$.

For $T \in V N(K), \epsilon>0$, choose $\alpha_{0}$, such that for $\alpha \geq \alpha_{0}$ and all $V \mid\left\langle v_{\alpha, V}^{\prime}, T\right\rangle$ $-\left\langle m^{\prime}, T\right\rangle \mid<\epsilon / 2$. Choose $(\alpha, V) \geq\left(\alpha_{.0}, V\right)$ such that $\left|\left\langle m, \phi^{*} T\right\rangle-\left\langle v_{\alpha, V}, \phi^{*} T\right\rangle\right|<$ $\epsilon / 2$. Then

$$
\begin{aligned}
\left|\left\langle m, \phi^{*} T\right\rangle-\left\langle m^{\prime}, T\right\rangle\right| \\
\quad \leq\left|\left\langle m, \phi^{*} T\right\rangle-\left\langle v_{a, V}, \phi^{*} T\right\rangle\right|+\left|\left\langle v_{a, V}, \phi^{*} T\right\rangle-\left\langle m^{\prime}, T\right\rangle\right| \\
\quad\left\langle\epsilon / 2+\left|\left\langle\phi v_{a, V}, T\right\rangle-\left\langle m^{\prime}, T\right\rangle\right|=\epsilon / 2+\left|\left\langle v_{a, V}^{\prime}, T\right\rangle-\left\langle m^{\prime}, T\right\rangle\right|<\epsilon .\right.
\end{aligned}
$$

Hence $\left\langle m, \rho^{*} T\right\rangle=\left\langle m^{\prime}, T\right\rangle$.

We can now prove our main result.

Theorem 11. If $G$ is nondiscrete, $V N(G)^{*}$ bas more than one invariant mean.

Proof. Let $A$ be a compact neighbourhood of $e$. The subgroup $K$ of $G$ generated by $A$ is an open subgroup and applying Proposition 10, we may already assume that $G$ is compactly generated. A theorem of Kakutani and Kodaira (see [5, Theorem 8.7]) now applies and we can find a compact normal subgroup $K$ such that $G / K$ has a countable basis for its open sets. If $G / K$ is discrete then $K$ is an open, compact nondiscrete subgroup of $G$ and by [2, Theorem 9] $V N(K)^{*}$ admits more than one invariant mean. But then by Proposition 10 again, so does $V N(G)$ *. We may therefore assume by Corollary 9 that $G$ has a countable basis for its open sets. Assume that there is a unique invariant mean $m$ on $V N(G)$. Let $\left\{V_{n}\right\}_{n=1}^{\infty}$ be a decreasing neighbourhood basis at $e$. For each $n$ choose $u_{n} \in P$ with spt $u_{n} \subset$ $V_{n}$. By Proposition $3,\left\{u_{n}\right\}$ is norm-convergent to invariance and by the proof of Theorem 4, each $w^{*}$-accumulation point of $\left\{u_{n}\right\}$ in $V N(G)^{*}$ is an invariant mean. It follows by the uniqueness of $m$ that $w^{*}-\lim u_{n}=m$. In other words $\left\{u_{n}\right\}$ is a weak Cauchy sequence in $A(G)$. But $A(G)$ is the predual of the von Neumann algebra $V N(G)$ so that a theorem of Sakai [7] applies to show that $A(G)$ is weakly sequentially complete. This implies that $m \in A(G)$. Proposition 5 now shows that $G$ is discrete and this is a contradiction. Applying Theorem 4 we see that $V N(G)$ must in fact have at least uncountably many invariant means.

We have already remarked that if $G$ is discrete then the unique invariant mean on $V N(G)$ is a faithful trace on $V N(G)$. At the other extreme we have

Theorem 12. If $G$ is nondiscrete and $m$ is an invariant mean on $V N(G)$ then $m(T)=0$ for all $T \in C_{\rho}^{*}(G)$. 
Remark. The algebra $C_{\rho}^{*}(G)$ is the $C^{*}$-completion of $L_{1}(G)$ acting by convolution on $L_{2}(G)$. If $G$ is amenable then $C_{\rho}^{*}(G)=C^{*}(G)$.

Proof. Since $L_{1}(G)$ is dense in $C_{\rho}^{*}(G)$ it suffices to show that $m\left(L_{f}\right)=0$ for all $f \in L_{1}(G)$.

Let $f \in L_{1}(G), \epsilon>0$. Let $U$ be a neighbourhood of $e$ such that $\int_{U}|f(g)| d g<\epsilon$ and choose $u \in P$ with spt $u \subset U$. Choose $\left\{u_{\alpha}\right\} \subset P$ such that $w^{*}-\lim u_{\alpha}=m$. Then $\left\{u_{\alpha}\right\}$ is $w$-convergent to invariance so that

$$
m\left(L_{f}\right)=\lim \int f(g) u_{\alpha}(g) d g=\lim \int f(g) u(g) u_{\alpha}(g) d g .
$$

But

$$
\begin{aligned}
\left|\int f(g) u(g) u_{a}(g) d g\right| & \leq \int|f(g)||u(g)|\left|u_{a}(g)\right| d g \\
& \leq \int_{U}|f(g)| d g<\epsilon \text { for all } \alpha .
\end{aligned}
$$

Hence $m\left(L_{f}\right)=0$.

\section{REFERENCES}

1. R. Arens, The adjoint of a bilinear operation, Proc. Amer. Math. Soc. 2 (1951), 839-848. MR 13, 659.

2. C. F. Dunkl and D. E. Ramirez, Existence and nonuniqueness of invariant means on $L^{\infty}(\hat{G})$ (to appear).

3. P. Eymard, L'algèbre de Fourier d'un groupe localement compact, Bull. Soc. Math. France 92 (1964), 181-236. MR 37 \#4208.

4. F. P. Greenleaf, Invariant means on topological groups and their applications, Van Nostrand Math. Studies, no. 16, Van Nostrand, Reinhold, New York, 1969. MR 40 \#4776.

5. E. Hewitt and K. A. Ross, Abstract harmonic analysis. Vol. 1: Structure of topological groups. Integration theory, group representations, Die Grundlehren der math. Wissenschaften, Band 115, Academic Press, New York; Springer-Verlag, Berlin, 1963. MR 28 \#158.

6. I. Namioka, Partially ordered linear topological spaces, Mem. Amer. Math. Soc. No. 24 (1957), 50 pp. MR 20 \#1193.

7. S. Sakai, On topological properties of $W^{*}$-algebras, Proc. Japan Acad. 33 (1957), 439-444. MR $20 \# 5437$.

DEPARTMENT OF MATHEMATICS, UNIVERSITY OF CANTERBURY, CHRISTCHURCH, NEW ZEALAND Canada

Current address: Department of Mathematics, University of Toronto, Toronto, Ontario, 